\title{
A NEW UPGRADING MODEL FOR DETECTING THE REACTION TO OBSTACLE APPEARANCE DURING WALKING USING EEG
}

\author{
Eduardo Iáñez, Álvaro Costa, Andrés Úbeda, Marisol Rodríguez-Ugarte, José M. Azorín \\ Brain-Machine Interface Systems Lab, Miguel Hernández University of Elche \\ Avda. de la Universidad s/n, Ed. Innova, 03202 Elche, Spain \\ eianez@umh.es, acosta@umh.es, aubeda@umh.es, maria.rodriguezu@umh.es, jm.azorin@umh.es
}

\begin{abstract}
In this paper a pseudo-online analysis has been performed to detect the reaction of both healthy users and incomplete Spinal Cord Injury patients to obstacle appearance during walking. A new methodology to improve model has been described. It consist of discarding trials where brain responses to the obstacles does not correspond with the expected. The results suggest that during the experiments performed with patients, distractions or delayed reactions decrease the results. By applying this methodology, results of patients increase more than $40 \%$ being similar to the obtained with healthy users.
\end{abstract}

Keywords: Obstacle appearance, EEG, model upgrading, environment changes.

\section{INTRODUCTION}

Nowadays, gait cycle is been analyzed in order to help in rehabilitation therapies [5]. This understanding can improve life of people that have lost walking ability by their rehabilitation or even helping them to completely recover the gait. To do that, robotic lower limb exoskeletons have significant potential for gait assistance and rehabilitation. To achieve a greater involvement of the patients, the use of a Brain-Machine Interface (BMI) can represent an important improvement.

Usually, patients are passive during their rehabilitation. Therefore, the study from cerebral information of the involvement of the patient in the therapy can improve the results of it $[7,4]$. Furthermore, detecting when the patient wants to starts or stop walking from EEG signals can generate commands control that allow controlling an exoskeleton.

Moreover, while a user is walking, other kind of potential can be analyzed, as the one produced when an unexpected obstacle appears [2]. In this case it is necessary to perform an emergency stop to avoid fall or collide. From cerebral signals is possible to detect the reaction of the user after perceiving the obstacle but before the user physically reacts.

In a previous work [8], it was identified the reaction of an user to an obstacle while walking. In a depth analysis of the EEG data it has been observed that part of the information used to create the model that allow identify this response does not correspond with the expected one. Therefore, the main goal of this work is the design of a new methodology that allows upgrade the model creation in order to improve the results. Four healthy users and four incomplete Spinal Cord Injury (i-SCI) patients performed the experiments. A pseudo-online analysis has been performed for both healthy and i-SCI patients.

This work is part of the BioMot project (Grant Agreement number IFP7-ICT-2013-10-611695) whose main goal is to analyze dynamic sensorimotor interactions in realistic human locomotion and design an artificial cognitive system for embodiment into bioinspired wearable assistive devices.

\section{MATERIAL AND METHODS}

This section describes the hardware and software architecture used for registering EEG signals and for analyzing the reaction of the user when obstacles appears randomly. Afterward, the protocol followed by users is explained. Then, features extraction method and a new upgrading model are described. Finally, the pseudo-online analysis used for obtaining the results is explained.

\subsection{Experimental Setup}

Fig. 1 shows the experimental environment where users perform the experiment. EEG signals have been acquired using a commercial amplifier ActiCHamp (Brainproducts) which has active electrodes that allows increasing the signal/noise ratio. The acquisition was performed using 31 electrodes with a sample frequency of $500 \mathrm{~Hz}$. The electrodes are distributed over the scalp in next positions: FCz, FC5, FC3, FC1, FC6, FC4, FC2, 
$\mathrm{Cz}, \mathrm{C} 5, \mathrm{C} 3, \mathrm{C} 1, \mathrm{C} 6, \mathrm{C} 4, \mathrm{C} 2, \mathrm{CPz}, \mathrm{CP} 5, \mathrm{CP} 3$, CP1, CP6, CP4, CP2, Pz, P3, P1, P4, P2, POz, $\mathrm{PO} 7, \mathrm{PO} 3, \mathrm{PO} 8$ and $\mathrm{PO} 4$. Ground was placed on $\mathrm{AFz}$ position and reference was taken as the average of electrodes TP9 and TP10 (mastoids) regarding to the 10/10 International System [3]. Matlab software is used for registering and data analyzing.

A treadmill Pro-form Performance 750 is used in order to achieve a constant velocity. A screen is placed in front of the treadmill. In this work an obstacle is defined as the background color change of the screen. When an obstacle appears, users must stop for a moment and then continue walking again.

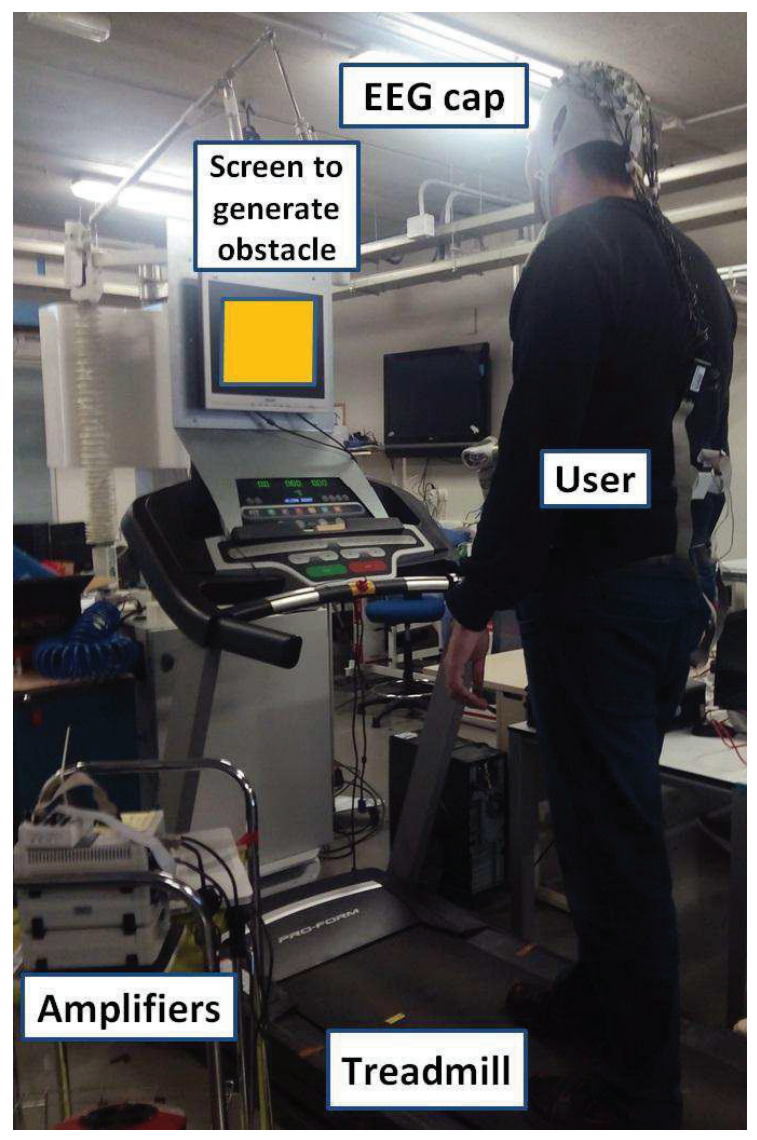

Figure 1: Environment where the user performs the experiments. The user walks on a treadmill while the screen shows the obstacles the user must react.

\subsection{Experimental procedure}

First, the equipment is prepared and the EEG cap is placed over the scalp. Conductive gel is used in order to reduce impedance and register a better quality EEG signals. Then, the user is asked to walk on a treadmill at $2 \mathrm{~km} / \mathrm{h}$ with 0 degrees of inclination. During each repetition, the user walks on the treadmill during 90 seconds while randomly obstacles appears. About 6-8 obstacles appear during a repetition. Users perform from 4 to 8 repetitions. When the user see that background color changes, he/she is asked to stop gait for a moment and then starts the gait again.

\subsection{Preprocessing}

First, registered signals of the 31 electrodes are studied. Then, 10 of them are discarded due to movement artifacts. They are located on the periphery on next positions: FC5, FC6, C5, C6, CP5, CP6, PO7, PO3, PO8 and PO4.

Later, windows of 0.5 seconds that correspond with the reaction of the user to the obstacle are selected and labeled as reaction class. These windows are in the range between 0.15 and $0.65 \mathrm{sec}-$ onds after the appearance of the obstacle according to previous studies [8]. Normal walking class belongs to user relaxation period. This class has the same number of 0.5 seconds windows as the previous one. An average EEG signal centered on an obstacle appearance of a healthy user and a patient is shown in Fig. 2. Windows taken for both classes are indicated.

After that, each EEG data window of the remaining 21 electrodes are bandpass filtered from 0.4 to $3 \mathrm{~Hz}$. To smooth brain activity contributions, the common average reference (CAR) is applied.

\subsection{Model upgrading}

The $70 \%$ of the data (normal walking and reaction windows classes) is processed to obtain the best features and to create a model. Windows with standard deviation higher than $40 \mu \mathrm{V}$ are rejected. The remaining data will be analyzed in pseudoonline.

Second column of Table 1 shows the total number of trials selected for the training data of both classes. In order to improve model creation a new methodology has been designed. This algorithm is based on discard trials of reaction class where the response is not as expected, i.e., when the targeted phenomena presents low or null differentiation. This way, the model is created with only suitable data and the results in the pseudo-online analysis can be improved.

During the experiment, user distraction or delayed reaction to the obstacle appearance could contribute to a bad model creation as part of the data do not correspond to the targeted phenomenon. This fact happen more frequently with patients. Therefore, brain responses of some of the registered trials does not match with the expected response. 


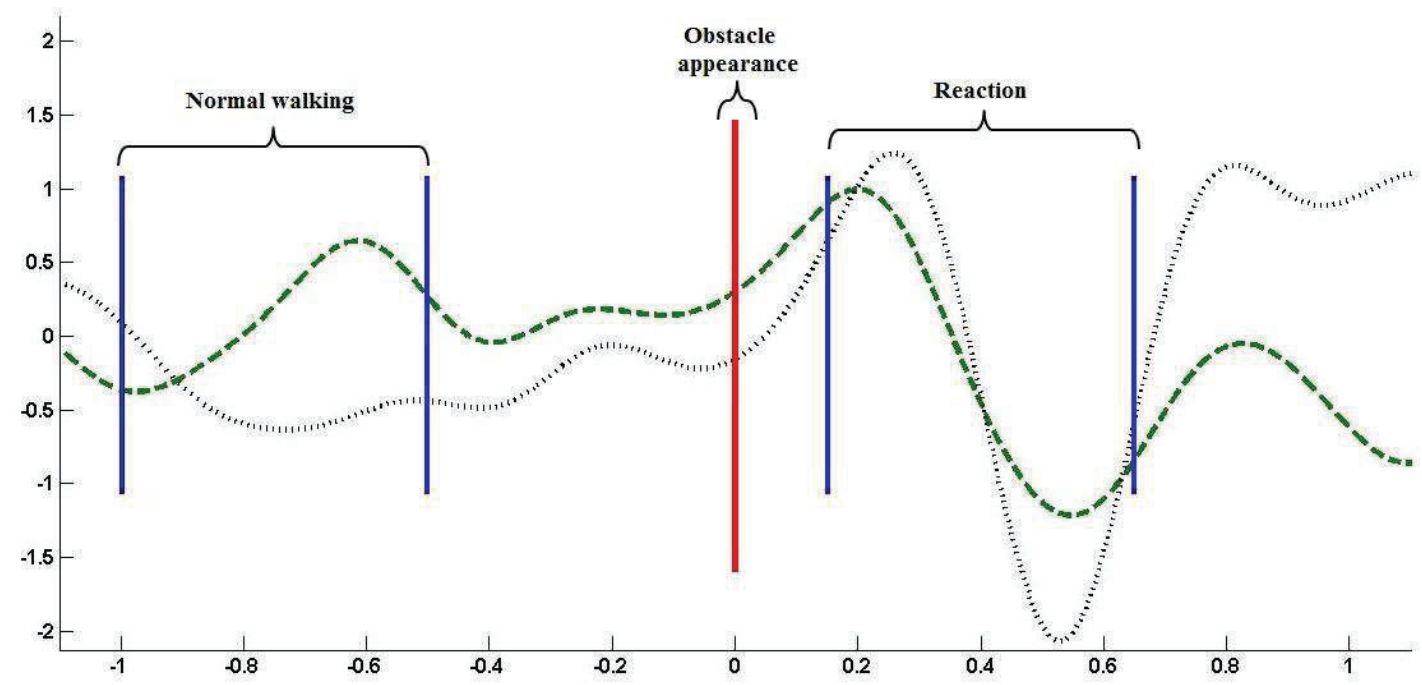

Figure 2: Example of the EEG signals registered on $\mathrm{Cz}$ channel in a healthy user and a patient after preprocessing. The graph shows an average of the trials for both subjects centered in at the moment the obstacle appears (red line). After the appearance of the obstacle a window of 0.5 seconds is selected as reaction class while a window of the same size previous to the obstacle is selected as normal walking class.
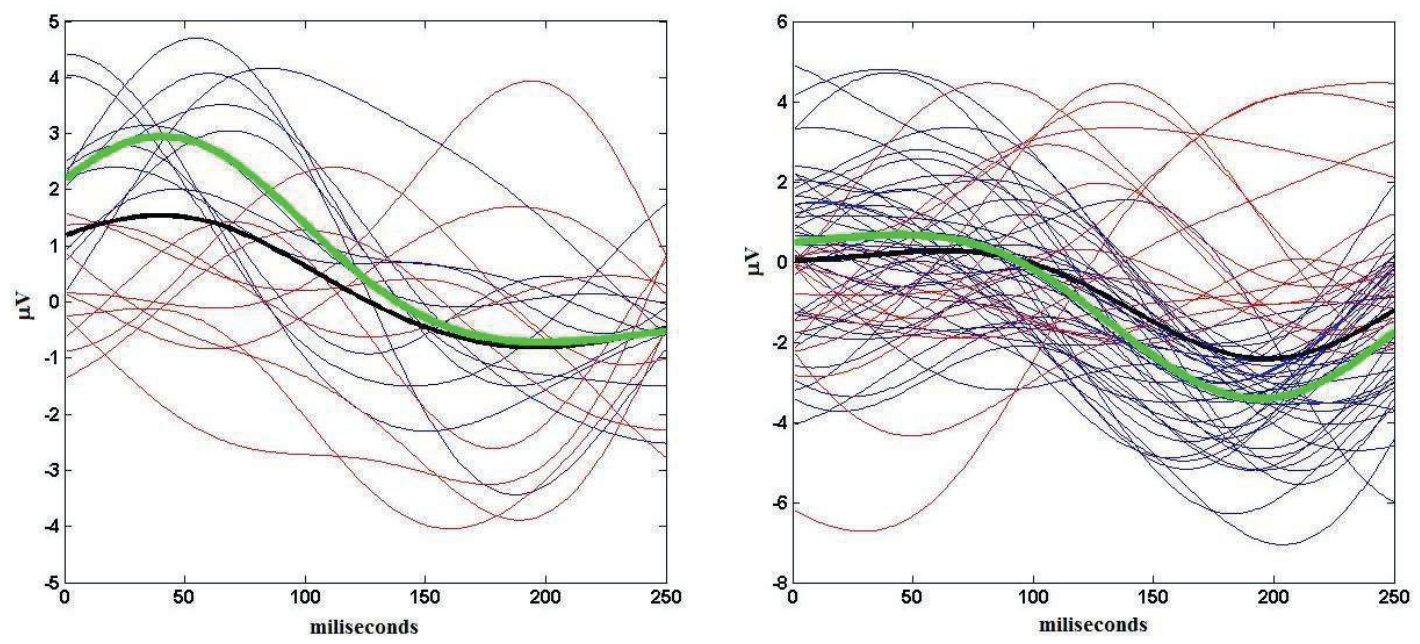

Figure 3: Example of the model upgrading for a healthy user (left) and a patient (right). The average signal before applying the method (thick black line) is emphasized after discarding several trials (thick green line). Groups of red lines are the ones to be discarded to create the model. Blue signals are used to create an upgrading model as they are more similar to the expected response. 
Table 1: Amount of trials used for creating the model before and after the upgrading. Last column indicates the number of trials included in the pseudo-online analysis.

\begin{tabular}{ccccc} 
User & $\begin{array}{c}\mathbf{N}^{\mathbf{o}} \text { of } \\
\text { total } \\
\text { trials } \\
\text { training }\end{array}$ & $\begin{array}{c}\mathbf{N}^{\mathbf{o}} \text { of } \\
\text { trials } \\
\text { after } \\
\text { upgrading }\end{array}$ & $\begin{array}{c}\text { \% of } \\
\text { valid } \\
\text { trials for } \\
\text { training }\end{array}$ & $\begin{array}{c}\mathbf{N}^{\mathbf{o}} \text { of } \\
\text { obstacles } \\
\text { that appear } \\
\text { pseudo-online }\end{array}$ \\
\hline H1 & 21 & 14 & 66,7 & 9 \\
H2 & 30 & 9 & 70,0 & 15 \\
H3 & 63 & 24 & 62,1 & 30 \\
H4 & 58 & 36 & 62,1 & 29 \\
\hline P1 & 28 & 22 & 76,9 & 12 \\
P2 & 28 & 14 & 50,0 & 12 \\
P3 & 54 & 35 & 64,8 & 22 \\
P4 & 58 & 41 & 70,7 & 30
\end{tabular}

The use of these wrong trials during the creation of the model can affect the final results. The methodology followed to select which of the training trials must be selected is the following:

- First, an average of all the reaction class trials is performed.

- Then, the similarity index between each trial and the averaged is obtained by a crosscorrelation [6].

- After analyzing several registers, it is assumed than less than a half of the data will correspond to incorrect trials.

- Afterward, the indices are split into two groups by using k-means clustering [9]: the most similar to the average and the remaining data.

- Finally, the trial with higher similarity to the average are used to obtain an improved averaged EEG response.

- The process is performed three times to ensure that all the trials do not correspond with the expected signal are discarded. After the first and the second iteration, the averaged model used to perform the cross-validation is obtained by using only the list of trials more similar to the previous averaged model.

The output of this process corresponds to the reaction class trials vector which should be used for creating the model.

Fig. 3 shows and example after the three iterations of a healthy user and a patient. The thick black line corresponds to the averaged signals taking into account all trials while thick green line correspond with the average after applying this process. The family of trials more similar to the expected signal are in blue while discarded trials are in red.

Table 1 shows the number of reaction class trials to be used after this method and the valid trials percentage for creating the model. An average of a $65.4 \%$ of the trials are kept to create the improved model.

Two models are created. The first one, includes all the reaction class trials and the second one, only the trials selected after the upgrading model method. Thereby, a comparison is performed.

\subsection{Processing}

In order to process each window (from normal walking or reaction class), only 6 main electrodes (FC1, FCz, FC2, C1, Cz and $\mathrm{C} 2$ ) are used. According to a previous analysis [8], these 6 electrodes show a more clear potential of a user reaction allowing to obtain the best results. The signal of the 6 electrodes are averaged. Then, the features vector is obtained by using a polynomial approach. The coefficients of a 5 th polynomial which best fits the average signal are selected. This implies that the features vector length is 6 samples per windows.

\subsection{Classifier}

A classifier based on linear discriminant analysis (LDA) has been used to differentiate between two classes of the features vector: reaction to the obstacle appearance versus normal walking [1]. Two models are created with the $70 \%$ of trials. The second one does not take into account several reaction class trials that are discarded after apply- 
Table 2: Comparison of $\mathrm{TP}$ and $\mathrm{FP} / \mathrm{min}$ results of healthy users and i-SCI patients when all trials are used to create the model versus when the upgrading model is used.

\begin{tabular}{ccccc} 
& \multicolumn{3}{c}{ Before improving model } & \multicolumn{3}{c}{ After improving model } \\
\cline { 2 - 5 } User & $\begin{array}{c}\mathrm{TP} \\
\text { obstacles }\end{array}$ & $\mathrm{FP} / \mathrm{min}$ & $\begin{array}{c}\mathrm{TP} \\
\text { obstacles }\end{array}$ & $\mathrm{FP} / \mathrm{min}$ \\
& 88,9 & 10,1 & 100,0 & 12,6 \\
H1 & 73,3 & 5,6 & 73,3 & 5,1 \\
H2 & 43,8 & 5,5 & 53,1 & 11,2 \\
H3 & 52,2 & 2,4 & 42,0 & 1,8 \\
H4 & 58,3 & 4,3 & 58,3 & 4,6 \\
Average & $\mathbf{6 4 , 6} \pm \mathbf{2 0 , 5}$ & $\mathbf{5 , 9} \pm \mathbf{3 , 2}$ & $\mathbf{6 7 , 1} \pm \mathbf{2 5 , 5}$ & $\mathbf{7 , 7} \pm \mathbf{5 , 1}$ \\
\hline \hline P1 & 0,0 & 0,1 & 100,0 & 6,6 \\
P2 & 36,9 & 4,9 & 54,2 & 8,1 \\
P3 & 20,5 & 1,2 & 69,2 & 7,1 \\
P4 & $\mathbf{5}$ & $\mathbf{2 , 6} \pm \mathbf{2 , 3}$ & $\mathbf{7 0 , 4} \pm \mathbf{2 0 , 7}$ & $\mathbf{6 , 6} \pm \mathbf{1 , 5}$
\end{tabular}

ing the method for upgrading the model creation. The results of applying both models in the pseudoonline analysis are compared.

\subsection{Pseudo-online analysis}

With the remaining 30\% test data, a pseudoonline analysis is performed. In this case, to simulate a real time analysis, the full EEG recording is segmented into windows of 0.5 seconds with an overlap of 0.4 seconds. Each window is preprocessed (bandpass and CAR filter) and processed (6 features obtained with polynomial approach). Then, in order to assign each window to reaction or normal walking class the model created is applied.

When a window coming from the reaction class is assigned to that class a true positive (TP) occurs. When the reaction class is detected and no obstacle has appear in the window a false positive (FP) is detected. If the FP are measured taking into account the total analyzed time a $\mathrm{FP} / \mathrm{min}$ are obtained.

Since the windows are overlapped, there are redundant information generating repeated reaction detections. To solve this, a correct detection is taken into account only if 2 consecutive windows are assigned to the reaction class. Last column of table 1 shows the number of obstacles that appear in the pseudo-online analysis.

\subsection{Users}

Four healthy users with ages between 23 and 34 years old, all male and right handed, took part on the experiments. Moreover, four incomplete SCI patients with ages between 34 and 49 years old, all male, three of them right handed, performed the experiments. All healthy and patients do not have any neurological disorder and with normal vision.

All subjects were informed and signed an informed consent according to Helsinski declaration. The experiments were approved by the Ethics Committee of the Miguel Hernández University of Elche (Spain).

\section{RESULTS AND DISCUSSION}

A pseudo-online analysis has been performed to obtain true positive and false positive per minute. The analysis have compared the results by creating a model with all the trials and with the improved model that discard trials where signal behavior are not the expected.

Table 2 shows the results for the 4 healthy users and the 4 i-SCI patients. The results obtained by healthy users when using all the trials for creating the model are around $65 \%$ of $\mathrm{TP}$ and $5.9 \mathrm{FP} / \mathrm{min}$. It is noticeable that there is not improvement in the results when the upgrading model is used. A Wilcoxon Sum-Rank test has been performed with a confidence interval of $98 \%$ to validate if there is a significant difference among the two groups of results [10]. This indicates that there is not significant difference in healthy users.

On the other hand, the results obtained by i-SCI patients before applying the upgrading model are too low. Despite FP/min are lower, the TP are about $29 \%$. In the case of patients the improved 
model substantially enhance the results. The TP increases more than $40 \%$ when all trials are used. $\mathrm{TP}$ and $\mathrm{FP} / \mathrm{min}$ results are similar with the obtained with healthy users. The Wilcoxon SumRank test applied show significant differences in these results.

This improvement could be due to the large amount of incorrect obstacle trials registered when i-SCI patients performed the experiments, either due to a low level of attention or by the difficulty to perform the experiment.

\section{CONCLUSION}

In this paper a pseudo-online analysis has been performed to detect the reaction of both healthy users and i-SCI patients to obstacle appearance. A new methodology to improve model has been described. It consist of discarding trials where brain responses to the obstacles does not correspond with the expected. The results suggest that during the experiments performed with patients, distractions or delayed reactions are performed which decrease TP results. By applying this methodology, results of patients increase more than $40 \%$ being similar to the obtained with healthy users.

In future works, new approaches that allow improve FP should be analyzed. Moreover, online experiments should be performed both healthy and i-SCI patients to validate the systems in a real environment. The obstacle appearance detected through EEG signals could be used as commands for exoskeletons. This way the exoskeleton can assist an stop of gait cycle if the user perceives an error.

\section{Acknowledgments}

This research has been supported by the European Commission 7th Framework Program as part of the project BioMot (FP7-ICT-2013-10, Grant Agreement no. 611695).

\section{References}

[1] Fukunaga, K., (2013) Introduction to Statistical Pattern Recognition. USA: Academic press, pp 131-153.

[2] Haefeli, J., Vögeli, S., Michel, J., Dietz, V., (2011) "Preparation and performance of obstacle steps: interaction between brain and spinal neuronal activity", Eur J Neurosci, vol $33(2)$, pp 338-48.

[3] Jasper, H.H., (1958) "The ten-twenty electrode system of the international federation". Electroencephalography and Clinical Neurophysiology, vol 10, pp 371-375.
[4] King, C.E., Wang, P.T., Chui, L.A., Do, A.H., Nenadic, Z., (2013) "Operation of a brain-computer interface walking simulator for individuals with spinal cord injury", $J$ Neuroeng Rehabil, vol 10, pp 77.

[5] Metzger, J.-C., Lambercy, O., Califfi, A., et al., (2014) "Assessment driven selection and adaptation of exercise difficulty in robotassisted therapy: a pilot study with a hand rehabilitation robot". Journal of neuroengineering and rehabilitation, vol 11, pp 154.

[6] Orfanidis, S.J., (1996) Optimum SignalProcessing. An Introduction. 2nd Edition, Prentice-Hall, Englewood Cliffs, NJ.

[7] Pfurtscheller, G., Muller-Putz, G.R., Scherer, R., Neuper, C., (2008) "Rehabilitation with brain-computer interface systems", Computer, vol 41, pp 58-65.

[8] Salazar-Varas, R., Costa, Á., Iáñez, E., Úbeda, A., Hortal, E., Azorín, J.M., (2015) "Analyzing EEG signals to detect unexpected obstacles during walking", Journal of NeuroEngineering and Rehabilitation, vol 12, pp 101.

[9] Spath, H., (1985) Cluster Dissectionand Analysis: Theory, FORTRAN Programs, Examples. Translated by J. Goldschmidt. New York: Halsted Press.

[10] Wilcoxon, F., Katti, S., Wilcox, R.A., (1970) "Critical values and probability levels for the wilcoxon rank sum test and the wilcoxon signed rank test", Selected tables in mathematical statistics, vol 1, pp 171-259. 